\title{
CONTRIBUCIÓN AL DESARROLLO DE LA BIOTECNOLOGÍA DESDE LA EDUCACIÓN EN LOS NIVELES DE LA BÁSICA Y MEDIA
}

Edgar Orlay Valbuena Ussa*

\begin{abstract}
The article presents the generalities of Biotechnology (definition, main applications and reaches), difficulties about its deficient development in Colombia. It proposed the realization of pedagogical projects with students enrolled on basic and middle education, with the goal to contribute to the solution about Natural Science teaching problems, and the development of Biotechnology in Colombia. A reflection is made about an experience with high school students on the frame of a pedagogical project that implied a work dealing contextualization and biotechnological applications.

Other elements are presented in general way to contribute to the development in Biotechnology from educational aspects.
\end{abstract}

\section{PALABRAS CLAVES}

Biotecnología, metabolismo, fermentación, cultivo de tejidos vegetales in vitro, anticuerpo monoclonal, biología molecular, ingeniería genética, organismos transgénicos

\section{INTRODUCCIÓN}

La biotecnología está constituida por las aplicaciones e innovaciones tecnológicas basadas en la utilización de sistemas biológicos ya sean: organismos, órganos, tejidos células o derivados del metabolismo, tendientes tanto a la creación de productos o procesos que representan bienes y/o servicios, como al desarrollo de investigación

Según el estado de desarrollo la biotecnología se puede categorizar en: áreas tradicionales (fermentaciones para la producción de bebidas, alimentos, antibióticos, ácidos orgánicos, glicerol, aminoácidos, proteína unicelular, etc.), y las nuevas biotecnologías (cultivos de tejidos, producción de anticuerpos monoclonales mapeo genético, la revolución del DNA recombinante, obtención de organismos transgénicos, técnicas de biología molecular tales como PCR, RFLPs, RAPDs. AFLPs. STRs, VNTRs,).

Es de anotar que la biotecnología ha tenido un gran impacto en la producción agropecuaria, industria química-farmacéutica, conversión de biomasa en energía,

\footnotetext{
* Departamento de Biología Universidad Pedagógica Nacional
} 
diagnóstico y tratamiento de enfermedades, control ambiental, conocimiento y conservación de la biodiversidad entre otros aspectos. Respecto a sus aplicaciones, bien merecen ser destacadas las relacionadas en los siguientes campos:

- La medicina principalmente en el diagnóstico y tratamiento de enfermedades genéticas, infecciosas, y/o parasitarias, en el transplante de órganos, la epidemiología, y la producción de vacunas y medicamentos, (DELPECH, 1993)

- La identificación humana a partir de la utilización de marcadores moleculares (principalmente alelos y repeticiones polimórficas), útiles en estudios de paternidad, medicina forense, criminalística, antropología y genética de poblaciones (PEREZ, et al. 1997, BUDOWLE B. et al. 1990).

- La medicina veterinaria y zootecnia: diagnóstico de enfermedades, producción de vacunas y medicamentos, mejoramiento animal, obtención de animales transgénicos, (AYCARDI, 1989).

- La agricultura: eliminación de virus, conservación de germoplasma, y micropropagación (mediante el cultivo de tejidos vegetales in vitro), fítomejoramiento, producción de bioinsecticidas, diagnóstico de enfermedades, obtención de plantas transgénicas (COLMAN, 1993, RENART, 1994),

- La industria: producción de alcohol, ácidos orgánicos, vinagre, productos lácteos, pan, biopolímeros, enzimas, aminoácidos, proteínas, metabolitos secundarios, antibióticos, hormonas, vacunas, antivirales, y otros fármacos, (MURRELL, 1993).

- El control ambiental: alternativas para la biorremediación, uso de microorganismos en el aprovechamiento de subproductos provenientes de la industria, utilización de microorganismos en el tratamiento de aguas residuales agroindustriales y/ó domésticas, aprovechamiento de los residuos lignocelulósicos, compostaje, manejo de desechos orgánicos con inóculos microbianos, degradación de hidrocarburos, fertilización de suelos, control biológico, y producción de polímeros biodegradables, entre otros. (DUBEY, 1993, CONGRESO MICROBIOLOGIA AMBIENTAL, 1997).

- La Biología de la Conservación: conocimiento (estudios de variabilidad genética utilizando marcadores bioquímicos, y/ó moleculares), y conservación (establecimiento de bancos de germoplasma, aportes para solución de problemáticas de impacto ambiental) de la biodiversidad (ROCA, 1991, CONGRESO MICROBIOLOGíA AMBIENTAL 1997, FAO/IPGR, 1994).

En relación con el alcance de la biotecnología, cabe anotar que a través de la Biología Molecular y de la Ingeniería Genética se ha llegado a un estado en el cual se puede manipular la información genética de los organismos proponiéndose procesos bioindustriales que constituyen una alternativa a los modos de producción tradicionales para acceder a la obtención de bienes y servicios. Por esto es lógico pensar que dicho proceso acelerado en las ciencias de la vida imponen nuevos modelos de desarrollo, convirtiéndose la biodiversidad en uno de los recursos más apreciados.

Como se puede analizar, la biotecnología tiene un gran impacto social y económico, y por su estado de desarrollo y aplicaciones, tiende a ser un elemento constitutivo de la cultura del siglo XXI. Sin embargo es necesario hacer educación en y a partir de ella, 
entre otras cosas por las implicaciones éticas que encierra la manipulación de organismos, o aún más: de su información genética.

\section{¿Por qué en Colombia a pesar de existir una gran biodiversidad está tan limitado el desarrollo de la biotecnología?}

Ahora bien, teniendo en cuenta que cualquier organismo representa potencialidades para las aplicaciones biotecnológicas, la riqueza en biodiversidad toma un gran valor, por cuanto representa multitud de posibilidades para la obtención de bienes y servicios en diversos campos. La alta diversidad biogeográfica y ecológica de Colombia, al igual que su gran riqueza de especies de muchas comunidades bióticas, colocan a nuestro país como uno de los que tiene una de la diversidad de especies más rica del mundo (ANDRADE, 1992). Infortunadamente debido al sometimiento de patrones culturales y económicos de utilización de la tierra y los recursos naturales, se ha generado una realidad de extinción masiva de especies. Para nadie es un secreto que los países más desarrollados que poseen el conocimiento científico y técnico, no cuentan con el recurso más valioso: la biodiversidad, este hecho está generando situaciones de tensiones a nivel político y social con las naciones como Colombia, ricas en diversidad biológica. Es así como según el tratado de la biodiversidad de Rio de Janeiro (CONFERENCIA NACIONES UNIDAS, 1992), los recursos genéticos se constituyen en prioridad de las naciones. En el marco de la política internacional Colombia ha adquirido varios compromisos, así existe la obligación de conservar la biodiversidad, y el diseño de una política de recursos genéticos que garantice la conservación, el enriquecimiento, y la accesibilidad de los mismos tanto para los colombianos como para los agentes externos del desarrollo económico, científico y tecnológico. (RESTREPO, 1994).

Por las ventajas comparativas de Colombia antes expuestas, se hace necesario además de conocer y conservar sus recursos genéticos, aprovecharlos para obtener beneficios a partir de ellos, sin causar un impacto negativo en el ambiente, una de las estrategias para hacerlo es mediante el uso de la biotecnología. Sin embargo el desarrollo de esta ciencia en nuestro país es pobre. En los países subdesarrollados la tendencia es que las aplicaciones biotecnológicas llegan a través de publicaciones y de recursos humanos formados en países industrializados, de allí que nuestra participación sea tangencial y con menores posibilidades para competir con el mercado internacional con la excepción de pocos grupos de investigadores como es el caso del liderado por el doctor Manuel Elkin Patarroyo.

Para el caso de Latinoamérica es notorio que las industrias no poseen tradición investigativa, y cuentan con escaso personal formado para crear y adaptar conocimientos, concentrándose en la producción de vinos y cervezas mediante una biotecnología tradicional. La producción de antibióticos, enzimas, aminoácidos y agroquímicos utiliza tecnologías importadas, salvo excepciones como en el caso de México, Brasil y Argentina. Dentro de los países latinoamericanos con mayor grado de desarrollo en biotecnología se destacan Cuba, México, Brasil y Argentina.

En el caso de Colombia existe un gran potencial de aprovechamiento de los recursos mediante procesos biotecnológicos. De acuerdo con la investigación realizada por el grupo de Biotecnología de la Universidad Nacional de Colombia (BUITRAGO, 1984), se detectaron grupos de investigación biotecnológica trabajando en las áreas de ingeniería genética: cultivo de tejidos; desarrollo de productos biológicos como vacunas y bioinsecticidas; producción de alcohol, alimentos, y ácido cítrico; y el uso de microorganismos en la fijación biológica del nitrógeno, sin embargo el desarrollo de estos 
procesos obedecía principalmente a trabajos de ingeniería. En relación con las industrias que hasta entonces utilizaban procesos biotecnológicas constituían cuatro sectores: productos biológicos (vacunas, sueros, etc.), industria alimentaria (quesos y leches ácidas), e industrias licoreras, y producción de materias primas, siendo las industrias de bebidas alcohólicas, alimenticias y de ácido cítrico las que representaban mayor rentabilidad.

Es evidente que en la última década han surgido nuevos grupos de investigación en biotecnología, con una infraestructura adecuada y con investigadores bien calificados, tanto en el sector oficial como en el privado incursionando incluso en el campo de la Biología Molecular y la Ingeniería Genética, sin embargo aún no es suficiente el grado de desarrollo alcanzado para poder aprovechar todo ó al menos parte del gran potencial de recursos con que cuenta nuestro país, y solucionar las problemáticas de producción, salud, y pérdida de biodiversidad. Como avances en el grado de desarrollo de esta ciencia en nuestro país conviene destacar los siguientes logros: la investigación comienza a alcanzar un carácter comercial (fitomejoramiento en cultivo de flores, banano, papa, café, producción de insumos, bioplaguicídas, biofertilizantes, etc.), se han implementado sistemas de diagnóstico de enfermedades (utilizando técnicas de biología molecular y celular) e instrumentos de prevención inmunológica, se están realizando estudios de identificación humana con un alto rigor (mediante la utilización de marcadores moleculares), se está produciendo conocimiento mediante investigaciones de punta (principalmente el trabajo del grupo del instituto de inmunología), se ha incursionado en la biotecnología ambiental con resultados interesantes, se está imponiendo la tendencia del sector privado (sectores agrícola, farmacéutico, de alimentos, y de saneamiento ambiental) a incorporar la biotecnología. Sin embargo perduran las problemáticas del escaso número de investigadores formados a alto nivel, y el hecho de que la mayoría de industrias se inclinan al uso de biotecnologías disponibles en el mercado (HODSON E., 1993).

A pesar de que, como ya se había mencionado, las aplicaciones biotecnológicas están introduciéndose como elemento cultural de la humanidad, cabe destacar como problemática la deficiente formación en lo que concierne los aspectos básicos de la Biotecnología, en la mayor parte de la población colombiana. Se hace así necesario el trabajo pedagógico desde los niveles de educación básica y media, para ello se requiere abordar la situación relacionada con el deficiente nivel de actualización de los docentes en ejercicio, máxime cuando los avances en esta ciencia son tan acelerados. Es de esperar que la mayoría de profesores de Ciencias Naturales y Educación Ambiental en ejercicio no conozcan los avances conceptuales y aplicaciones en biotecnología. En un estudio realizado con un grupo de 26 profesores de Ciencias Naturales del Distrito Capital se evidenció que en más del $50 \%$ se presentaban dificultades a nivel conceptual en temáticas tales como: manipulación genética, técnicas de marcación de moléculas, marcadores moleculares, mapeo genético, sondas de ADN, donación y tecnología del ADN recombinante; también en más de la mitad de la población estudiada se manifestó que tan solo algunas veces o nunca accedían a información de actualización en biotecnología (BECERRA y MOJICA, 1996).

\footnotetext{
¿Por qué a través de la biotecnología se puede mejorar la enseñanza de las ciencias, contribuyendo a su vez al desarrollo de la biotecnología?
} 
A través de la investigación, se ha visto que la educación que se implementa en muchas instituciones, generalmente conlleva al estudiante a seguir un esquema repetitivo de contenidos, desconociendo su realidad y sus intereses, sin lograr generar realmente un aprendizaje significativo. Además, es evidente que existen limitaciones para que el estudiante tanto de educación básica como de media, por una parte acceda al conocimiento científico a partir del lenguaje duro", como suele ocurrir en el esquema de enseñanza tradicional donde se abusa de las verdades absolutas, y además que pueda aplicar dichos conocimientos a través de cambias actitudinales. Otra problemática en la enseñanza de las ciencias radica en que el profesor, en su afán de transmitir conocimientos, y tras alcanzar conclusiones correctas de la temática que se esté trabajando en clase, es el único que las obtiene. Se hace necesario entonces innovar estrategias donde a partir de resolución de problemas generados desde las necesidades e intereses del mundo de la vida, y mediante planteamiento y desarrollo de proyectos que involucren lo práctico, sea posible generar en los estudiantes conocimiento científico mediante un proceso de descubrimiento y desarrollo de sus potencialidades investigativas, sin descuidar las aplicaciones e implicaciones que este hecho tiene para el avance de a ciencia y la tecnología de Colombia Como lo plantea Ocampo 1993) el desarrollo económico de un país está muy en relación con la educación científica requiriéndose mejorar este aspecto de la formación en todos los niveles de educación, fundamentalmente para que la población pueda asimilar los principios científicos y familiarizarse con el campo de la tecnología aplicada al trabajo productivo y a las necesidades de la economía nacional.

Para el desarrollo científico y tecnológico de un país se hace necesario formar en este sentido a grupos preferiblemente desde los niveles de educación básica y media, esto implica trabajar tanto en el aspecto cognitivo, como en el del desarrollo de actitudes científico-tecnológicas, desde luego haciendo un esfuerzo por vincular este tipo de trabajos a las vivencias de los estudiantes, más concretamente haciendo una concientizacíón de la importancia de estos campos en la resolución de problemas y el desarrollo del país. Para alcanzar este propósito es necesario ofrecer una formación básica a la población juvenil en que se prepare para asumir una época donde el desarrollo de una nación depende en gran medida de la capacidad de producir conocimiento y tecnología.

\section{La propuesta}

Se propone generar y desarrollar proyectos pedagógicos con estudiantes de los niveles de educación básica secundaria y media, pretendiendo, a partir de la implementación de aplicaciones biotecnológicas, contribuir a la solución de problemáticas a nivel local, regional, y/o nacional. Aunque a través de estos trabajos realizados con estudiantes de bachillerato no es posible generar innovaciones biotecnológicas, existe la intencionalidad de aportar en la valoración aprovechamiento y uso, tanto racional como ético, de los recursos genéticos, la formación en valores, el desarrollo de las capacidades comunicativas, el descubrimiento y desarrollo de potencialidades de actitudes científicas, la concientización de la importancia del avance de la biotecnología: el aprendizaje significativo de Ciencias Naturales, y de la Biología en particular, a partir de la problematización de procesos, tendiendo a integrar los proyectos a situaciones generadas por las necesidades e intereses desde el mundo de la vida: y por todo lo anterior en el desarrollo humano integral, y de la biotecnología 
Además de los anteriores elementos de la propuesta, también se está trabajando en tres frentes más: la formación de docentes con una visión general de la biotecnología, y en particular, de alguna de sus ramas en el caso de profundizar la investigación en el área de la Biotecnología; y la socialización de resultados, y producción de material tanto didáctico como divulgativo de las generalidades y particularidades de la biotecnología.

\section{Principales logros alcanzados}

A partir del trabajo realizado al rededor de la técnica de cultivo de tejidos vegetales in vitro con estudiantes del nivel de básica secundaria de varios colegios de Santafé de Bogotá, consistente básicamente en una fase de familiarización y contextualización tanto con el trabajo en laboratorio como con la técnica en si, seguida de una fase de trabajo por proyectos y mediante investigación cualitativa se han podido establecer como principales tendencias en los escolares:

- Problematización conceptual nutrición relación estructura-función. totipotencialidad celular, reproducción, intercambio gaseoso, transporte de fluidos, clasificación de compuestos químicos y de organismos microorganismos, funciones celulares, herencia, biodiversidad, cálculos matemáticos).

- Establecimiento de jerarquización e interrelaciones entre conceptos biológicos

- Entender el manejo de variables y condiciones experimentales en el proceso de estructuración de un diseño.

- Sistematización, reflexión, y análisis de resultados, y contextualización frente a un marco conceptual

- Manifestación de actitudes de curiosidad científica.

- Adquisición de destrezas en el manejo de instrumental, preparación de soluciones químicas, y trabajo de laboratorio en general atendiendo a las normas de bioseguridad

- Proposición proyectos de aplicación resultado de la necesidad de aplicar la técnica para la resolución de problemas cercanos ( $p$. ej. introducción in vítro de algunas especies de importancia del páramo, comportamiento del lulo ante diferentes reguladores de crecimiento, propagación in vitro de limón como alternativa ante un problema de ataque de una plaga a esta especie, entre otros).

- Adquisición de un lenguaje técnico de la aplicación biotecnológica (el cual se utiliza con relativa propiedad), y hasta cierto punto de terminología científica.

- Explicar con claridad las aplicaciones de la técnica de cultivo de tejidos vegetales in vitro

- Formación de actitudes de disciplina de trabajo.

- Manifestación de motivación y preferencia hacía las actividades lúdicas y de prácticas de laboratorio 
- Valoración del trabajo e incremento de la autoestima (por la apropiación de experimentos generados desde ellos).

- Valoración en cuanto a los recursos genéticos y a la importancia de la ciencia.

- Desarrollo de actitudes de tolerancia y capacidad argumentativa en el momento de hacer las discusiones.

- Posibilitar en algunos la manifestación de expresiones artísticas mediante el dibujo, o simplemente la contemplación de lo estético, de lo vivo ( $p$ ej. en morfologías de plantas, tejidos, células, o colonias de microorganismos).

- Formación de actitudes éticas en cuanto al manejo y aprovechamiento de los recursos genéticos, valoración de la importancia de la biodiversidad, las políticas y el desarrollo de la ciencia para el desarrollo social y económico de un país.

- Manifestación de interés hacia el conocimiento y conservación de la biodiversidad,

- Manifestación de actitudes de camaradería, respeto y familiaridad con compañeros y profesor.

- Persistencia de la actitud de renuencia.

- Actitud de cierta resistencia a realizar la actividad de talleres.

A partir del análisis de estos resultados se reflexiona que el propósito de la enseñanza de las Ciencias, en el área de la Biología en este caso, más que transmitir contenidos es permitir al estudiante las posibilidades, orientación, medios y recursos para que pueda comprender procesos y conceptos y así pueda dar explicaciones a las problemáticas detectadas durante el trabajo académico, concibiendo al educando en sus diversas e interrelacionadas dimensiones: como ser social, cultural, humano, con intereses y principios éticos propias.

Es de destacar que la modalidad de trabajo implementada en el proyecto pedagógico genera un ambiente educativo diferente del que generalmente vive el estudiante, en su relación con: el conocimiento, sus compañeros, su profesor, y sus actividades académicas: hecho que posibilita que el trabajo trascienda de lo conceptual a la formación teniendo en cuenta el desarrollo humano integral, y los aspectos sociales y éticos.

Retomando el tema de las actitudes científicas, éstas se entienden no como un conjunto de creencias y predisposiciones de acción de una forma irreflexiva, sino por el contrario involucra posiciones críticas en relación con pertinencia y limites respecto a un problema, de esta forma implica que la persona no se imita a obedecer órdenes, y responde a la necesidad de acudir a varias disciplinas para solucionar los problemas. A partir de la reflexión de los resultados, además de las anteriores, también se consideran actitudes científicas: la curiosidad; la creatividad; la disciplina de trabajo; el alto nivel de sistematización de información, de análisis, de sustentación, de discusión, y de detección y formulación tanto de problemas como de alternativas de solución. Mockus (1989) caracteriza la actitud científica por una movilidad reflexivamente regulada entre distintos "juegos de lenguaje" reconocidos en su especificidad y en su ámbito de validez, que implica elegir reflexivamente el modo en que se miran las cosas y el modo en que éstas 
se dicen, y por ende la coherencia entre la forma como se preparan las acciones y como se actúa. Implicando una forma consciente en la elección teniendo en cuenta los riesgos, limites y falibilidad. Como ya se había planteado inicialmente, el desarrollo de estas actitudes contribuye con la formación de futuros si no investigadores, por lo menos sí individuos reflexivos y críticos, con elementos básicos para disminuir el fenómeno de que la población quede a la merced de las decisiones de la minoría de las expertos; todo esto a la postre directa ó indirectamente va a tener un impacto en el desarrollo de la Ciencia y la Tecnología en el país.

Por otra parte, de acuerdo con lo planteado por Ocampo (1993), el currículo más que incluir un cúmulo de contenidos obligatorios, debe tender a la aproximación a los conocimientos básicos necesarios para abordar la educación científica. Acorde con esto, y con las políticas educativas nacionales vigentes, en relación con la flexibilidad curricular, el trabajo realizado hasta ahora pretende, más que introducir progresivamente contenidos en biotecnología en los programas de Ciencias Naturales y Educación Ambiental, integrar aplicaciones biotecnológicas como elementos constitutivos a los Proyectos Educativos Institucionales, a través del trabajo con los estudiantes interesados, quienes además de crecer en su formación académica y personal, se orientan en cuanto a su futuro profesional.

Cabe reflexionar que el país necesita consolidar grupos de investigación altamente calificados, que can seguridad se van a desarrollar más fácil y rápidamente si desde la educación básica y media se hace una exploración de intereses y aptitudes hacia el conocimiento científico y en el caso especifico que nos ocupa, de la biotecnología.

Finalmente, y de acuerdo con lo planteado como propuesta, en los otros aspectos se ha avanzado: respecto a la formación de docentes con una visión general de la biotecnología, por un lado se ha aportado desde el trabajo con las futuros profesores en el actual programa de Licenciatura en Biología de la Universidad Pedagógica Nacional, a través de la realización de cursos electivos con profundización en el área, el desarrollo de trabajos de grado en el campo en cuestión, y el desarrollo de proyectos pedagógicos dentro de la práctica docente, involucrando aspectos biotecnológicos, por otra parte se diseñó el Programa de Formación Permanente de Docentes Educación en Biotecnología en los Niveles de Básica y Media del Distrito Capital En cuanto a la investigación en el área de la biotecnología, se encuentran en estado de ejecución los proyectos "Introducción in vitro de Espeletia spp.", y Aislamiento y caracterización bioquímica de cepas de Bacillus thuringiensis". Por último en lo referente a socialización y producción de material escrito, al internar del proyecto se han generado los materiales. "Algunos aspectos de Biología Molecular, y sus Aplicaciones Biotecnológicas" (dirigido a docentes de Ciencias Naturales y Educación Ambiental en ejercicio", Los microorganismos en la industria alimenticia" (dirigido a estudiantes de básica secundaria y media), "Manual para realización de prácticas de Cultivo de Tejidos Vegetales in vítro" (dirigido a estudiantes de básica secundaria y medía), y el manual teórico práctico: «Aprendamos Biología Molecular» (dirigido a estudiantes de educación media).

\section{BIBLIOGRAFÍA}


ANDRADE G., RUIZ. J. P., GÓMEZ, R. Biodiversidad y uso de recursos naturales. FESCOL CEREC, Bogotá 1992.

AYCARDI, Eduardo. "Biotecnología moderna y productividad animal". En: Revista Universidad Nacional. No. 9 (mar-abr. 1989).

BECERRA, Edgar: MOJICA Pedro. "Diseño de un curso de actualización en el área de Ciencias Naturales con énfasis en aspectos de Biología Molecular y sus aplicaciones biotecnológicas dirigido a docentes de educación básica secundaria”. Trabajo de grado, Departamento de Biología, Universidad Pedagógica Nacional. 1996.

BUDOWLE, B.; WAYE, J.: SHUTLER, G.; BAECHTEL, F. E. Restriction endonuclease for restriction fragment lenght polymorphism analysis of biological evidence samples. J. Forens. Sci. No. 35, págs. 530-536, 1990.

BUITRAGO, G., et al. Diagnóstico de la biotecnología en Colombia. Bogotá. Informe a Colciencias, 1984.

COLCIENCIAS. "Tecnologías para la vida y el desarrollo". 1993.

COLMAN, Alan. "Aplicaciones comerciales de la Ingeniería genética: Perspectivas y logros". En: MURREL y ROBERTS. Introducción a la Ingeniería Genética. México. De Limusa, 1993.

CONFERENCIA DE LAS NACIONES UNIDAS SOBRE EL MEDIO AMBIENTE Y EL DESALLO. Rio de Janeiro, 3 al 14 de junio de 1992. FESCOL, 1992.

CONGRESO DE MICROBIOLOGIA AMBIENTAL, Memorias. Pontificia Universidad Javeriana, 1997

DELPECH, Marc. "Un nuevo instrumento en el diagnóstico médico", En: Mundo científico. Vol. 13. No. 132 (feb. 1993); pp. 172-182.

DUBEY, P.C. A text book of Biotechnology. S. Chand \& Company Ltd. 1993.

FAO/IPGP Normas para bancos de genes. Informe Anual. 1994.

HODSON, Elizabeth En: COLCIENCIAS "Biotecnologías de la vida para el desarrollo". Santafé de Bogotá D.C., tercer mundo editores, 1993.

HUGHES, K, W "Ornamental species”, en: Conquer. B.V. Edit Cloning agricultural plants vía in vitro techniques CRC Press, Bocca Raton, Florida, USA. 1981.

MONTOYA. Dolly. Biotecnología: Presente y futuro para Colombia y América Latina

MOCKUS, Antanas. (1989). "Formación básica y actitudes científicas" En: Educación y Cultura. No. 17, pp 11-16. Bogotá. Universidad Nacional de Colombia. Instituto de Biotecnología. 1990.

MURRELL y ROBERTS. Introducción a la ingeniería genética. México. De. Limusa, 1993. 
OCAMPO, José Fernando (1993). "La enseñanza de las Ciencias Naturales en la educación primaria y secundaria" En: Educación y Cultura, No. 30, pp. 5-10.

PEREA, Margarita. "Biotecnología agrícola. La nueva revolución verde". En: Revista Universidad Nacional de Colombia. Marabr. 1989, No. 10.

PEREZ, Lezaun, et al. Microsatellite variation and the differentiation of modem humans. Hum. Genet., No. 99, pags. 1-7, 1997.

ROCA, M. William.; MROGINSKI, Luis A. Cultivo de tejidos en la agricultura.

Fundamentos y aplicaciones. Cali-Colombia. Centro Internacional de Agricultura Tropical CIAT. 1991.

RENART, Miguel. Avances en ingeniería genética. Madrid. Consejo Superior de Investigaciones Científicas, 1994.

RESTREPO, Miguel. "Biodiversidad, Biotecnología y patentación de vida. En: Esteros. Vol. 2, Nos. 5-6 (abr.- sep. 1994), pp. 26-33. 